\title{
A Novel ELF Radar for Major Oil Deposits
}

\author{
Jamesina J. Simpson, Student Member, IEEE, and Allen Taflove, Fellow, IEEE
}

\begin{abstract}
This letter proposes a novel extremely low frequency (ELF) radar for major oil deposits. Using our recently developed whole-Earth electromagnetic wave propagation model based upon the finite-difference time-domain method, we have determined that detection of the radial (vertical) component of the scattered $\boldsymbol{H}$-field provides a sensitive means to detect oil fields that are located within several kilometers of the Earth's surface. As an example, we provide numerical simulations of ELF radar returns from a hypothetical Alaskan oil field excited by a 20-Hz pulse emitted from the former U.S. Navy site in Wisconsin. The proposed method would potentially provide means to rapidly and inexpensively conduct aerial surveys of thousands of square kilometers for significant oil deposits.
\end{abstract}

Index Terms-Aerial electromagnetic surveying, Earth, electromagnetic sounding, extremely low frequency (ELF), finite-difference time-domain (FDTD), oil field, SQUID magnetometer, U.S. Navy Wisconsin Transmitter Facility (WTF).

\section{INTRODUCTION}

D EEP electromagnetic (EM) sounding of the Earth using controlled sources has been employed for decades as a means to determine the electrical properties of the Earth's lithosphere. Previously, controlled sources such as power lines, electrified railroads, and pulsed magnetohydrodynamic (MHD) generators have been employed for such probing [1]. However, the data obtained from these methods exhibits an unsatisfactory wide variance [1]. Further, the above techniques can only be applied at considerable cost and effort, making their implementation infeasible in many regions of the world. As a result, new approaches to deep EM geophysical prospecting are desirable. If a superior technique applicable on a global scale were developed, it could permit both rapid and inexpensive surveying of the entire Earth's crust. Such a method would be of particular interest for locating anomalous conductivity structures such as mineral deposits and major oil fields.

Since the early 1990s, a promising new system for global remote sensing has been under investigation [1], [2]. This technique involves the use of a powerful radio-transmitting antenna in the extremely low frequency (ELF) $(3 \mathrm{~Hz}$ to $3 \mathrm{kHz}$ ) range. Velikhov et al. [1] outlined the following advantages of employing ELF radio waves up to $100 \mathrm{~Hz}$ for EM sounding:

1) sufficiently large skin depth for deep probing (100 $\mathrm{m}$ in ocean and 10-15 km in crystalline shields);

2) low propagation attenuation, which could provide global remote sensing of the Earth with a single source;

Manuscript received April 19, 2005; revised June 26, 2005. This work was supported in part by the National Computational Science Alliance under Grant DMS040006N.

The authors are with the Electrical Engineering and Computer Science Department, Northwestern University, Technological Institute, Evanston, IL 60208 USA (e-mail: j-simpson@northwestern.edu).

Digital Object Identifier 10.1109/LGRS.2005.856118
3) good stability compared to higher frequency methods that are more influenced by ionospheric disturbances.

Due to these particular properties of ELF waves, Velikhov et al. introduced the idea of simultaneously performing studies around the globe involving oil-bearing rock, seismic regions, ionospheric wave propagation, and other areas of geophysics using one powerful ELF source.

The ELF sounding methodology described in [1] and [2], however, involves EM field impedance measurements to determine the resistivity of the underlying rock. This requires measurement of both the tangential electric field $E_{\text {tan }}$ and the tangential magnetic field $H_{\tan }$ near the Earth's surface. In this letter, we propose a novel ELF radar for major oil deposits that requires detection of only the radial magnetic field, $H_{r}$, as a low-frequency pulse is radiated outward from a distant powerful ELF antenna. Using rigorous, large-scale computational solutions of Maxwell's equations, we have determined that $H_{r}$ exhibits an unexpected and very high degree of sensitivity to the presence of deeply buried conductivity anomalies of the lithosphere, much more so than $H_{\text {tan }}$. We propose exploiting this phenomenon to establish a means to rapidly and inexpensively conduct aerial surveys of thousands of square kilometers for significant oil fields [3].

As an example of the capabilities of our proposed ELF radar, we report numerical simulations of ELF radar returns from a hypothetical Alaskan oil field excited by a $20-\mathrm{Hz}$ pulse emitted from the former U.S. Navy site in Wisconsin. For this study, we use our recently developed whole-Earth EM wave propagation model [4], [5] based upon the finite-difference time-domain (FDTD) solution of Maxwell's equations [6]. This technique permits a direct three-dimensional (3-D) time-domain calculation of round-the-world ELF propagation accounting for arbitrary horizontal as well as vertical geometrical and electrical inhomogeneities/ anisotropies of the excitation, ionosphere, lithosphere, and oceans. The robustness of the FDTD method has in recent years caused it to become a popular tool for analyzing EM wave propagation around the complete Earth-ionosphere waveguide [7]-[9].

\section{ThreE-Dimensional LATITUDE-LONGITUDE FDTD GRID}

Fig. 1 illustrates the general layout of the FDTD space-lattice as seen from the transverse magnetic (TM) plane at a constant radial coordinate [5]. The lattice is a logically Cartesian $2 M \times M \times K$-cell arrangement, where $M$ is a power of 2 . We see that the grid cells follow lines of constant latitude, $\theta=$ constant, where $\theta$ is the usual spherical angle measured from the north pole; and along lines of constant longitude, $\phi=$ constant, where $\phi$ is the usual spherical azimuthal angle measured from a specified prime meridian. In this manner, each TM plane of the grid shown in Fig. 1 is comprised of isosceles trapezoidal cells away 


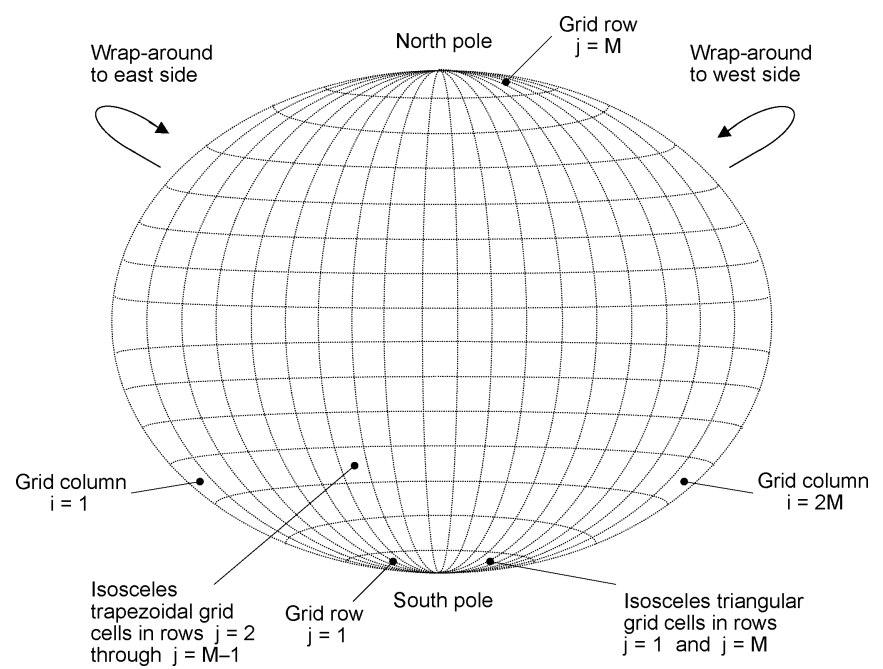

Fig. 1. General layout of the 3-D FDTD lattice covering the complete Earth-sphere as seen in a TM plane at a constant radial coordinate.

from the north and south poles [5], and isosceles triangular cells at the poles [5]. Similarly, each transverse electric (TE) plane at a constant radial coordinate is comprised of isosceles trapezoidal cells away from the north and south poles, and a polygon cell at each pole.

We choose to have the same angular increment in latitude $\Delta \theta=\pi / M$ for each cell in the grid. Thus, the south-north span of each trapezoidal or triangular grid cell is $\Delta_{s-n}=\pi R / M$, where $R$ is the radial distance from the center of the Earth. To maintain square or nearly square grid cells near the equator, we select the baseline value of the angular increment in longitude $\Delta \phi$, to equal $\Delta \theta$. However, this causes the west-east span of each cell $\Delta_{w-e}=R \Delta \phi \sin \theta$ to be a function of $\theta$. This could be troublesome for cells near the north and south poles where $\theta \rightarrow 0$ and $\theta \rightarrow \pi$, respectively. There, the geometrical eccentricity of each cell $\Delta_{s-n} / \Delta_{w-e}=\Delta \theta /(\Delta \phi \sin \theta)$ would become quite large, and the numerical stability and efficiency of the FDTD algorithm would be degraded. We mitigate this problem by merging pairs of adjacent cells of the TM plane in the west-east direction, effectively halving the cell eccentricity [5]. This process can be repeated several times as the grid approaches a pole, allowing the user to specify a maximum allowable cell eccentricity.

The wrap-around or joining of the east and west edges of the lattice occurs along a specific line of constant longitude, or meridian. This joining is, in effect, a periodic boundary condition applied at each $j$-row of lattice cells, whether trapezoids or triangles [5].

Given the above assumptions, Ampere's Law in integral form can be applied to develop an FDTD time-stepping relation for each electric $E$ field components of the grid [5]. Similarly, Faraday's Law in integral form can be applied to develop an FDTD time-stepping relation for the magnetic $H$ field components of the grid [5].

\section{Details of The FDTD EM Sounding Study at ELF}

In this letter, our goal is to determine how the presence of a major oil field influences the surface $H$-field components when excited by an ELF pulse radiated from a distant transmitter. Specifically, we model the oil field as a lithosphere conductivity anomaly of lateral dimensions $28 \times 39 \mathrm{~km}$ and thickness $1.25 \mathrm{~km}$ located in Alaska at $156^{\circ} \mathrm{W}, 69^{\circ} \mathrm{N}$. The anomaly is assumed to have a conductivity that is ten times lower than that of the surrounding strata. By way of comparison, Alaska's Kuparuk River oil field spans a lateral area of about $1000 \mathrm{~km}^{2}$ [10].

We use two separate FDTD models to calculate the potential ELF radar signatures of all three $H$-field components at a point $P$ directly above the conductivity anomaly. Model A serves as the reference (i.e., no conductivity anomaly present). Model B includes the conductivity anomaly assumed to be located at a median depth of $1.25 \mathrm{~km}$.

For the remainder of the global lithosphere model, we utilize topographic and bathymetric data from the NOAA-NGDC "Global Relief CD-ROM."' These data are mapped onto the 3-D space lattices of both models with an assumed lateral resolution of $40 \times 40 \mathrm{~km}$ at the equator. The complete FDTD grid extends to a depth of $100 \mathrm{~km}$ into the lithosphere and to an altitude of $100 \mathrm{~km}$. The grid is terminated at both edges in the radial direction by a perfect electric conductor (PEC). The PEC boundary is adequate for this model since the skin depth within the lithosphere and reflection height of the ionosphere are each less than $100 \mathrm{~km}$. Subgridding [6] is used to refine the radial grid resolution in the lithosphere near the Earth's surface to $1.25 \mathrm{~km}$, a factor of four times finer than the nominal radial resolution of $5 \mathrm{~km}$ used in the atmosphere and deeper within the lithosphere. Numerical experiments have shown that the subgridding introduces sufficiently low numerical error relative to the physical phenomena being modeled. For the lithosphere, conductivity values are assigned according to [11], depending upon the location of an E component (i.e., below an ocean or within a continent). For the atmosphere, both day- and nighttime exponential conductivity profiles used in [12] are assumed, with midnight occurring at GMT. The effective waveguide height of reflection for these profiles is roughly $48 \mathrm{~km}$ for day and $76 \mathrm{~km}$ for night [12].

Velikhov et al. [1] and Bashkuev and Khaptanov [2] employed "Zevs," an ELF antenna system located in the north of the Kola Peninsula in Russia, for their impedance studies. Aside from Zevs, the only known practical ELF communication system is the facility operated by the U.S. Navy in upper Michigan and Wisconsin until September 2004 [13]. Our computational model assumes the Wisconsin Transmitting Facility (WTF) section of the Navy site located near Clam Lake, WI $\left(90.9^{\circ} \mathrm{W}, 46.5^{\circ} \mathrm{N}\right)$ as the ELF source used to detect the lithosphere conductivity anomaly in Alaska (at a distance of about $4.4 \mathrm{Mm}$ ). We assume nominal operation of this facility with two orthogonal ground lines, each $22.5 \mathrm{~km}$ long and carrying a current of $300 \mathrm{~A}$, one oriented in the north-south direction and the other in the east-west direction. However, balancing between the skin depth in the lithosphere and the radiation efficiency of WTF, our assumed carrier frequency is $20 \mathrm{~Hz}$, rather than the $75-\mathrm{Hz}$ Navy frequency. We further assume that the transmitted signal is a pulse that is generated by double-sideband amplitude modulation of the $20-\mathrm{Hz}$ carrier by

\footnotetext{
${ }^{1}$ See http://www.ngdc.noaa.gov/mgg/fliers/93mgg01.html.
} 


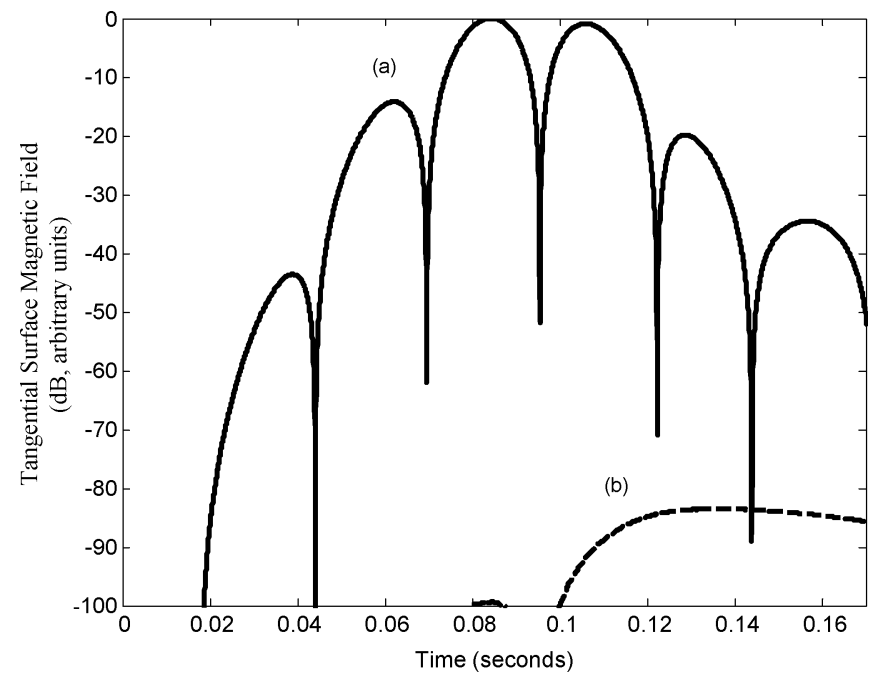

Fig. 2. Comparison of the absolute value of the time waveforms for surface $H_{\text {tan }}$ at $156^{\circ} \mathrm{W}, 69^{\circ} \mathrm{N}$ in Alaska for a pulsed $20-\mathrm{Hz}$ signal originating from WTF, normalized to the peak incident $H_{\text {tan }}$ : (a) Incident pulse and (b) radar signature (the difference between the calculated surface magnetic field component in models A and B) for a $28 \times 39 \times 1.25 \mathrm{~km}$ conductivity anomaly at a mean depth of $1.25 \mathrm{~km}$. The Matlab function "smooth" was applied to the data to filter out high-frequency fluctuations arising from subtraction noise.

a Gaussian envelope waveform of full-width at half-maximum $42.5 \mathrm{~ms}$. Below the WTF, we model the Laurentian Plateau (or Canadian Shield), a large region of low-conductivity rock of $2.4 \mathrm{E}-4 \mathrm{~S} / \mathrm{m}$ that extends northward from the Great Lakes toward the Arctic Ocean and includes much of Canada and Greenland.

\section{Computational Modeling Results}

Using a decibel scale, Fig. 2 shows our calculated results for the absolute value of the time-waveform of the surface $H_{\tan }$ at observation point $P$ normalized to the incident $H_{\mathrm{tan}}$. The following two cases are illustrated: the incident pulse (Model A, no conductivity anomaly present [Fig. 2(a)] and a radar signature (the difference between the calculated surface magnetic field component in models A and B) for the $28 \times 39 \times 1.25 \mathrm{~km}$ conductivity anomaly at a median depth of $1.25 \mathrm{~km}$ [Fig. 2(b)]. We see that the $H_{\tan }$ radar signature of the conductivity anomaly is well below $-80 \mathrm{~dB}$ relative to the incident pulse. Therefore, although the incident pulse is detectable, given the experience of the Navy's virtually worldwide communications with its deeply submerged submarines, the presence of the conductive anomaly in the lithosphere introduces only a very small perturbation in the surface $H_{\tan }$ and is hence difficult to detect.

Similar to Fig. 2, Fig. 3 shows the corresponding calculated results for the absolute value of the time-waveform of the surface $H_{r}$ at observation point $P$ normalized to the incident $H_{r}$. Here, we see that the $H_{r}$ radar signature of the conductivity anomaly rises to $-15 \mathrm{~dB}$ relative to the incident $H_{r}$. Therefore, the presence of the conductivity anomaly in the lithosphere introduces a very large perturbation in $H_{r}$ at the surface, which greatly bolsters the possibility of detecting its presence over $H_{\tan }$. In fact, since $H_{\tan }$ is required for the impedance measurement technique reprted in [1] and [2], comparing Figs. 2 and 3

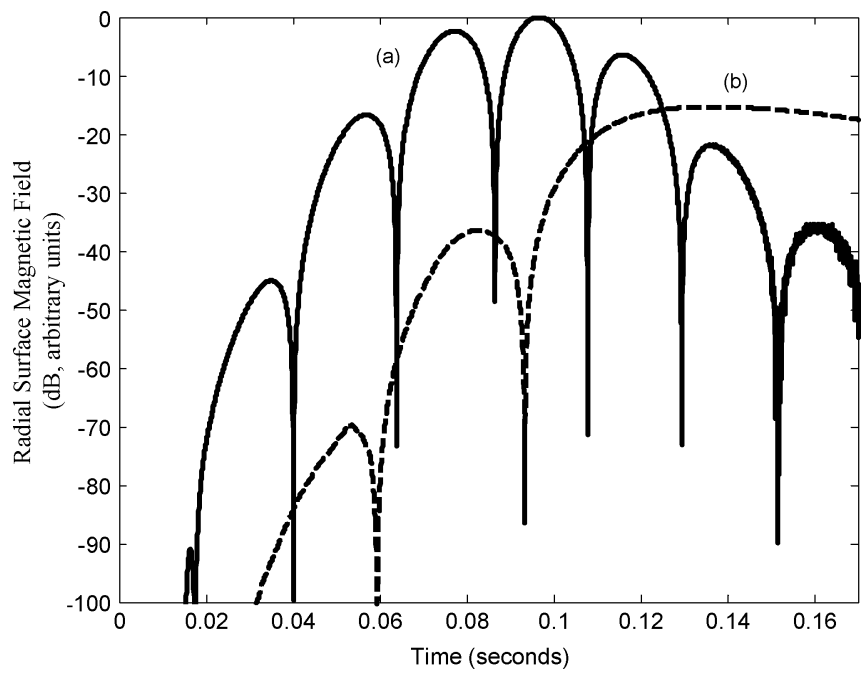

Fig. 3. Comparison of the absolute value of the time waveforms for surface $H_{r}$ at $156^{\circ} \mathrm{W}, 69^{\circ} \mathrm{N}$ in Alaska for a pulsed $20-\mathrm{Hz}$ signal originating from WTF, normalized to the peak incident $H_{r}$ : (a) Incident pulse and (b) radar signature (the difference between the calculated surface magnetic field component in models A and B) for a $28 \times 39 \times 1.25 \mathrm{~km}$ conductivity anomaly at a mean depth of $1.25 \mathrm{~km}$. The Matlab function "smooth" was applied to the data to filter out high-frequency fluctuations arising from subtraction noise.

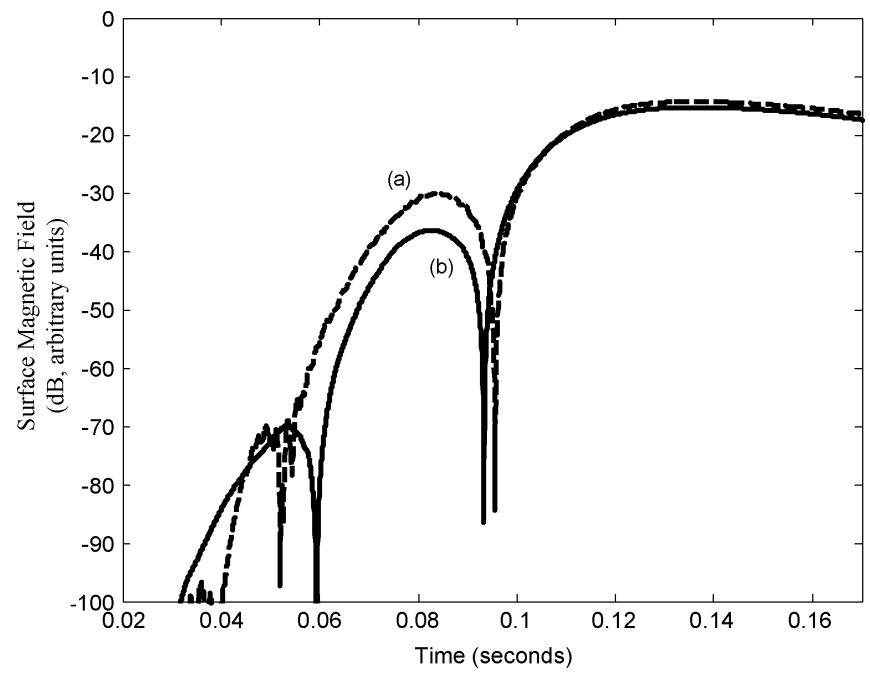

Fig. 4. Comparison of the absolute value of the radar signature time waveforms of Figs. 2 and 3 for a $28 \times 39 \times 1.25 \mathrm{~km}$ conductivity anomaly at a mean depth of $1.25 \mathrm{~km}$ at $156^{\circ} \mathrm{W}, 69^{\circ} \mathrm{N}$ in Alaska. Both are normalized to the peak incident $H_{r}$ for a pulsed 20-Hz signal originating from the WTF: (a) $H_{\tan }$ and (b) $H_{r}$. The Matlab function "smooth" was applied to the data to filter out high-frequency fluctuations arising from subtraction noise.

makes clear that the sensitivity of the impedance technique is considerably less than afforded by a measurement of $H_{r}$.

Fig. 4 shows the (b) curves of Figs. 2 and 3, the calculated time-waveforms of the surface $H_{\tan }$ and $H_{r}$ radar signatures, in a different form. Here, by normalizing both waveforms to the incident $H_{r}$, we see that the presence of a $1.25-\mathrm{km}$ median-depth conductivity anomaly generates $H_{\mathrm{tan}}$ and $H_{r}$ radar signatures of comparable magnitudes. Therefore, considering the results of Figs. 2 and 3 , the perturbation produced by the anomaly relative to the background $H_{r}$ is over $65 \mathrm{~dB}$ larger than that for $H_{\mathrm{tan}}$. We believe that this relatively enhanced radial magnetic field component is due to looping currents within the lithosphere that circulate around the thin conductivity anomaly in the horizontal 
plane. We also find from analogous studies involving deeper conductivity anomalies that measuring $H_{r}$ becomes increasingly superior for anomalies at greater depths. Furthermore, the outline (shape and size) of conductivity anomalies can be determined. Finally, we find that these $H_{r}$ radar signatures are detectable given the results of [2] in which $H_{\tan }$ was measured for impedance variation studies at distances of $4 \mathrm{Mm}$ from the ELF source and for depths of 10-20 km.

\section{IMPLiCATIONS FOR A Possible DeteCtion SyStem}

Our FDTD calculations have shown that a powerful distant ELF antenna can be used to locate oil-bearing rock within several kilometers of the Earth's surface by measuring the highly sensitive surface $H_{r}$. Given these results, we propose that an ELF source such as the WTF, used in conjunction with an airborne magnetometer having sufficient sensitivity to $H_{r}$, can be used to rapidly and inexpensively detect oil fields over thousands of square kilometers. In fact, considering the very low propagation attenuation of EM waves at ELF [5], employing a sufficiently powerful ELF antenna permits use of this sounding technique on a global scale.

We propose the use of SQUID magnetometers for the aerial surveys described in this letter because they: 1) measure the magnetic field directly; 2) offer higher sensitivity at frequencies less than $150 \mathrm{~Hz}$ than other receiver technologies; and 3) provide higher bandwidths [14]. Further, they have already been successfully implemented in aerial surveys [14].

\section{CONCLUSION AND ONGOING WORK}

In this letter, we have proposed a novel ELF radar for major oil deposits. As an example of this new technology, we provided numerical simulations of ELF radar returns from a hypothetical Alaskan oil field excited by a $20-\mathrm{Hz}$ pulse emitted from the former U.S. Navy site in Wisconsin. By employing our 3-D FDTD model of the entire Earth-ionosphere cavity, we determined that the surface $H_{r}$ exhibits an unexpected and very high degree of sensitivity to the presence of deeply buried conductivity anomalies of the lithosphere, much more so than $H_{\text {tan }}$. We therefore proposed that airborne SQUID magnetometers recording only $H_{r}$, synchronized with the transmission of an ELF pulse from a distant, powerful ELF antenna, may potentially provide the global locations of oil fields in a rapid and inexpensive manner. Although the case studied here is at $4.4 \mathrm{Mm}$ from the transmitter, we believe that detection at much larger distances should be possible because at $20 \mathrm{~Hz}$ the additional attenuation is only about $0.4 \mathrm{~dB} / 4 \mathrm{Mm}$, which should be well within the dynamic range of existing instruments.
Our ongoing work in this area includes developing a parallelized alternative hexagonal/pentagonal 3-D geodesic FDTD grid with superior efficiency [15], [16].

\section{REFERENCES}

[1] E. P. Velikhov, A. A. Zhamaletdinov, A. N. Shevtsov, A. D. Tokarev, Y. M. Kononov, L. B. Pesin, G. M. Kadyshevich, M. I. Pertel, and A V. Veshchev, "Deep electromagnetic studies with the use of powerful ELF radio installations," Isvestiya, Phys. Solid Earth, vol. 34, no. 8, pp. 615-632, 1998.

[2] Y. B. Bashkuev and V. B. Khaptanov, "Deep radio impedance sounding of the crust using the electromagnetic field of a VLF radio installation," Izvestiya, Phys. Solid Earth, vol. 37, no. 2, pp. 157-165, 2001.

[3] J. J. Simpson and A. Taflove, "Whole Earth FDTD modeling of ELF electromagnetic sounding of oil deposits with the U.S. Navy's ELF transmitter system," presented at the USNC/URSI National Radio Science Meeting, Washington, D.C., Jul. 2005.

[4] — "Two-dimensional FDTD model of antipodal ELF propagation and Schumann resonance of the Earth," IEEE Antennas Wireless Propagat. Lett., vol. 1, pp. 53-56, 2002.

[5] - "Three-dimensional FDTD modeling of impulsive ELF propagation about the Earth-sphere," IEEE Trans. Antennas Propagat., vol. 52, no. 2, pp. 443-451, Feb. 2004.

[6] A. Taflove and S. C. Hagness, Computational Electrodynamics: The Finite-Difference Time-Domain Method, 3rd ed. Norwood, MA: Artech House, 2005.

[7] Y. Ando, M. Hayakawa, A. V. Shvets, and A. P. Nickolaenko, "Finite difference analyzes of Schumann resonance and reconstruction of lightning distribution," Radio Sci., vol. 38, no. 6, p. 1103, 2003. DOI: 10.1029/2002RS002752.

[8] A. Soriano, E. A. Navarro, D. L. Paul, J. A. Porti, J. A. Morente, and I. J. Craddock, "Finite-difference time domain simulation of the Earth-ionosphere resonant cavity: Schumann resonances," IEEE Trans. Antennas Propagat., vol. 53, no. 4, pp. 1535-1541, Apr. 2005.

[9] H. Yang and V. P. Pasko, "Three-dimensional finite difference time domain modeling of the Earth-ionosphere cavity resonances," Geophys. Res. Lett., vol. 32, no. L03114, 2005. DOI:10.1029/2004GL021343.

[10] U. S. Congress, Office Technol. Assessment, "Oil production in the Arctic National Wildlife Refuge: The technology and the Alaskan oil context," U.S. Gov. Printing Office, Washington, DC, OTA-E-394, Feb. 1989.

[11] J. Hermance, "Electrical conductivity of the crust and mantle," in Global Earth Physics: A Handbook of Physical Constants. Washington, DC: AGU, 1995.

[12] P. Bannister, "The determination of representative ionospheric conductivity parameters for ELF propagation in the Earth-ionosphere waveguide," Radio Sci., vol. 20, no. 4, pp. 977-984, 1985.

[13] J. Garza, "Navy pulls plug on embattled ELF site," Milwaukie J. Sentinel, Sep. 17, 2004. [Online]. Available: www.jsonline.com.

[14] J. B. Lee, D. L. Dart, R. J. Turner, M. A. Downey, A. Maddever, G. Panjkovic, C. P. Foley, K. E. Leslie, R. Binks, C. Lewis, and W. Murray, "Airborne TEM surveying with a SQUID magnetometer sensor," Geophysics, vol. 67, no. 2, pp. 468-477, Mar.-Apr. 2002.

[15] J. J. Simpson and A. Taflove, "Efficient modeling of impulsive ELF antipodal propagation about the Earth sphere using an optimized two-dimensional geodesic FDTD grid," IEEE Antennas Wireless Propagat. Lett., vol. 3, pp. 215-218, 2004.

[16] - "3-D FDTD modeling of ULF/ELF propagation within the global Earth-ionosphere cavity using an optimized geodesic grid," presented at the IEEE AP-S Int. Symp., Washington, DC, Jul. 2005. 